\title{
Erosion of inferior vena caval filter noted during robotic assisted laparoscopic partial nephrectomy
}

\author{
Ryan C. Hutchinson, David D. Thiel, Todd C. Igel \\ Department of Urology, Mayo Clinic, Jacksonville, Florida, United States
}

\section{ABSTRACT}

Inferior Vena Cava (IVC) filters are mechanical devices implanted to provide prophylaxis against pulmonary emboli in patients for whom standard anticoagulation is either inadequate or contraindicated. A 67-year-old female with a 10-year-old indwelling IVC filter underwent robotic assisted laparoscopic partial nephrectomy for a right upper pole renal mass. Renal hilum dissection was complicated by adhesions secondary to eroded IVC filter struts. IVC filter erosion is a well-described phoenomena in both the radiologic and surgical literature. As many as $25 \%$ of filters are noted to be radiographically eroded; however, the incidence of clinically significant erosion is much less. Given the placement of endovascularly delivered IVC filters in close proximity to many urologic operative fields, it is important for urologists to be aware of the potential of eroded devices when pursuing para-caval dissections.

\section{BACKGROUND}

A 67-year-old female with a history of recurrent deep vein thrombosis on warfarin presented with an incidentally identified $1 \mathrm{~cm}$ enhancing upper pole right renal mass and stress type urinary incontinence. She had previously undergone laparoscopic cholecystectomy, appendectomy, and placement of a Simon Nitinol IVC filter. Options, including surgical intervention and surveil- lance, were discussed, and she elected to pursue a combined robotic assisted laparoscopic partial nephrectomy with simultaneous transbobturator urethral sling placement. Pre-operative imaging noted her previously placed IVC filter near the region of the renal veins (Figure-1).

During the renal hilar dissection, dense adhesions surrounding protruding prongs from her previously placed IVC filter were noted in the region of the gonadal vein and renal vein (Figure-2). Adhesion around the prongs made mobilization of the duodenum more difficult than usual. Careful dissection was used to free up the protruding prongs (Figure-2). Once the prongs were isolated, the renal artery and vein were able

Figure 1 - CT scan image of IVC filter.

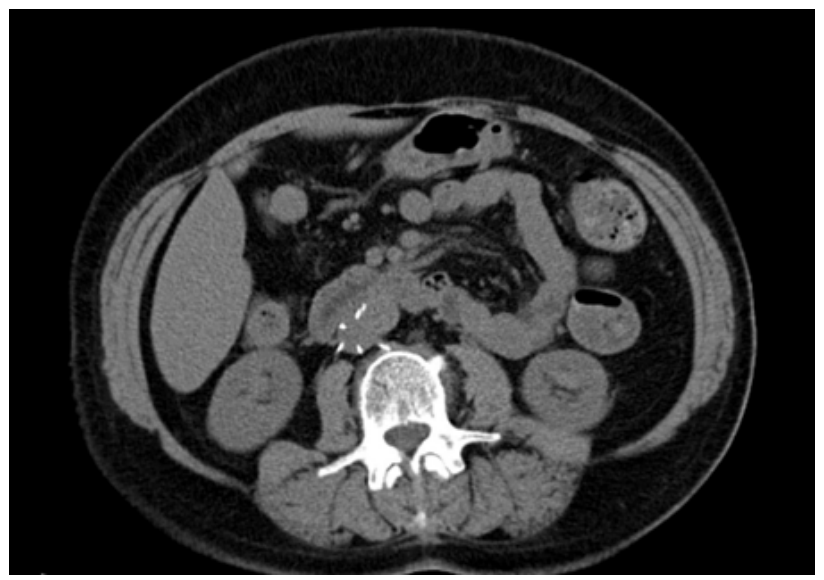

This CT image notes an IVC filter in the region of the gonadal vein and renal veins. Protruding prongs can be noted posteriorly. 
Figure 2 - Intra-operative image of the IVC prongs.

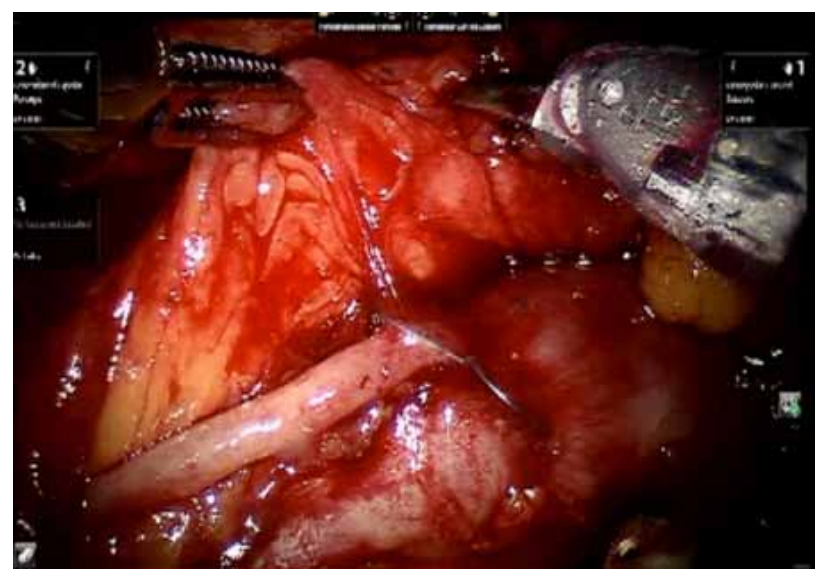

2 metal prongs are noted protruding through the IVC near the gonadal vein and renal vein. These prongs were carefully dissected free of their attachments in order to minimize interfere with duodenum or renal vein dissection.

to be circumferentially dissected in anticipation of renal hilum clamping prior to mass excision. The patient then underwent standard partial nephrectomy. Following partial nephrectomy, there was no further manipulation of the filter prongs. The final pathology was consistent with hemangioma. The patient tolerated the procedure well and was discharged after an uneventful postoperative course.

\section{DISCUSSION}

The Inferior Vena Caval (IVC) filter is a mechanical prophylactic option for patients at high risk for thromboembolic complications where medical anticoagulation is contraindicated or inadequate to control risk (1). Since Greenfield's seminal paper in 1973, they have been a part of the surgical armamentarium to prevent perioperative complications (1). Currently placed endovascularly through femoral or jugular venous access, these filters offer a low peri-procedural complication rate. However, the long-term implications of an indwelling foreign body within the vena cava are less well characterized and can present operatively challenging pathology (2). We present the case of an eroded inferior vena caval strut discovered incidentally during robotically assisted laparoscopic partial nephrectomy.
Erosion of inferior vena caval filters is not rare with as many as one in four (25\%) demonstrating radiographic evidence of erosion. Clinically, these erosions are typically asymptomatic, with rates of symptomaticity reported in less than $1 \%$ of known filters (2). Given the anatomic location of these filters adjacent to the aorta, spine, renal hilum, portal vein, and duodenum, there is the potential for serious complication from protruding struts. Pain, ureteral injury, aortic perforation, intracardiac migration, and duodenal perforation have all been reported as late complications of indwelling IVC filters (2).

There is often disparity between CT scan appearance of IVC prong protrusion and actual appearance intra-operatively. Examination of the fat planes surrounding the vena cava may hold more valuable information. Visualization of the fat planes around the IVC can be predictive of prong protrusion that may cause adhesion or bleeding during surgery. A perfect example of this is in our Figure-1. There is evidence of protrusion of an IVC prong posteriorly on CT scan but the fat planes are in-tact. One pronge near the renal vein anterior-lateral has no surrounding fat plane, hence possibly predictive that there might have been some bleeding causing adhesions.

Newer filters are sometimes equipped with a mechanism allowing for later retrieval in situations where thrombotic risk is temporary, as in the perioperative period or after trauma. Retrievable IVC filters can offer the advantage of a non-permanent prophylaxis and are retrievable in approximately $90 \%$ of attempted retrievals (3). This contrasts sharply with actual reported rates of retrieval of 2-41\%; this disparity appears to persist even under thorough monitoring protocols and appears multifactorial, related to overall medical condition, ongoing indication for filter, patient preference and patient volition (4). Also, while rare, clinically significant erosions can be devastating and require multiple high-morbidity procedures to correct. Currently controversy exists as to the risks, benefits and indications for IVC filters; level one evidence will be needed to further inform this discussion (5). 
To our knowledge, no guidelines exist for management of incidentally discovered eroded IVC struts. Management options for incidentally identified eroded struts include clipping protruding struts flush with the IVC wall in order to dull sharp edges and prevent viscous perforation, buffering with external surgical devices, and no action.

\section{REFERENCES}

1. Greenfield LJ, McCurdy JR, Brown PP, Elkins RC: A new intracaval filter permitting continued flow and resolution of emboli. Surgery. 1973; 73: 599-606.

2. Streiff MB: Vena caval filters: a comprehensive review. Blood. 2000; 95: 3669-77.

3. Stein PD, Alnas M, Skaf E, Kayali F, Siddiqui T, Olson RE, et al.: Outcome and complications of retrievable inferior vena cava filters. Am J Cardiol. 2004; 94: 1090-3.
4. Zakhary EM, Elmore JR, Galt SW, Franklin DP: Optional filters in trauma patients: can retrieval rates be improved? Ann Vasc Surg. 2008; 22: 627-34.

5. Becher RD, Corriere MA, Edwards MS, Godshall CJ: Late erosion of a prophylactic Celect IVC filter into the aorta, right renal artery, and duodenal wall. J Vasc Surg. 2010; 52: 1041-4.

\footnotetext{
Correspondence address: Dr. David D. Thiel Mayo Clinic Florida Department of Urology Davis Building 3E 4500 San Pablo Rd Jacksonville, FL, 32224, USA Fax: +1 904 953-2218 E-mail: thiel.david@mayo.edu
} 